\title{
ANÁLISIS DE LA RESOLUCIÓN DE PROBLEMAS COMO ESTRATEGIA DE ENSENANZA DE LA BIOLOGÍA
}

\author{
SIGÜENZA, A.F. y SÁEZ, M.J. \\ Departamento de Biología Celular y Farmacología, Valladolid.
}

\section{SUMMARY}

Solving-problems as a biology teaching strategy could make the process of comprehension easier and lead to the student's acquisition of knowledge. This paper analyses problem-solving as a basic element of the above strategy (method). For this reason it first proposes the definition of the terms "problem» and «problem-solving» inside practical teaching and secondly discusses the nature of solving as a method within the particular case of biology teaching-learning.

\section{INTRODUCCIÓN}

Tradicionalmente, los conocimientos de la biología se han contemplado y se han transmitido como una colección de hechos, principios, leyes, reglas e interacciones lógicas. Sin embargo, este tipo de enseñanza es considerada, por algunos autores, inferior (Stenhouse $1987 \mathrm{p}$. 120) si se la compara con aquélla que induce a los estudiantes al conocimiento, teniendo como propósito la comprensión. Además, actualmente la rápida evolución que está sufriendo esta disciplina la convierte en una ciencia muy dinámica donde continuamente surgen problemas y preguntas de interés tanto científico como social, cuya solución puede restultar muy difícil. En este contexto, la enseñanza de la biología, a nuestro juicio, requiere el uso de estrategias que faciliten la comprensión y capaciten al alumno para la resolución de problemas. La comprensión es, sin duđa, el objetivo principal de la enseñanza y aunque resulte problemático epistemológicamente conceptualizar quées loque constituye la comprensión respecto de cualquier área de conocimiento, podremos evidenciarla por la capacidad de operar bien conforme a unos criterios, seleccionando de forma adecuada información, estrategias, algoritmos, etc., para un fin propio, es decir, por la capacidad de resolver problemas.

De hecho, desde mediados de los años setenta, el desarollo curricular en su preocupación por los procesos de la enseñanza de las ciencias experimentales señala la resolución de problemas como proceso clave en la educación (Dewey 1975, Garrett 1987). El uso de problemas se considera un componente vital de la enseñanza de las ciencias (Reif 1981, Maskill y Wallis 1982, Harlen 1983, Garrett 1988).

En este nuevo marco de la enseñanza están surgiendo trabajos en el área de la resolución de problemas que aconsejan qué «problemas» pueden ser utilizados y cómo deben ser organizados (Healey 1980, Philpot y Sellwood 1983, Gil y Martínez-Tortegrosa 1987, Kempa 1986) y el papel de la resolución de problemas en la estrategia de enseñanza (Frazer 1982, Garrett 1986).

En la actualidad no resuita sorprendente observar el gran interés que los profesores de ciencias experimentales 
manifiestan hacia la resolución de problemas, quizás motivado por el mejor conocimiento de los procesos del pensamiento y del aprendizaje humanos. Sin embargo, resulta difícil aún establecer el significado más preciso del término y los aspectos docentes implicados en la teoría y práctica de la resolución de problemas, lo que probablemente se vea favorecido por la carencia de una teoría unificada en este sentido (Smith 1988).

En consecuencia, se está manifestando la necesidad de desarrollar tentativas dinámicas organizadas para posibilitar al alumno el acceso al conocimiento. En este sentido, ta resolución de problemas como instrumento de cambio metodológico podría contribuir a ello.

Este trabajo pretende, en primer lugar, encontrar una defínición del término problema, que corresponda al sentido de éste en la realidad del aula dentro del marco de la enseñanza de la biología, y en segundo Iugar, intenta mostrar la coherencia del modelo de resolución de problemas como estrategia de enseñanza de la biología, con la construcción de conocimiento que tiene lugar en cada sujeto, individualmente, para acceder a la comprensión, destacando la importancia que tiene el hecho de que el problema sea definido por los alumnos en la propia aula. Con ello, esperamos contribuir a la elaboración đe una teoría unificada de la resolución de problemas como estrategia de enseñanza de las ciencias en general y como instrumento de cambio metodológico.

\section{DEFINICION DE TÉRMINOS}

En ocasiones resulta difícil aceptar una definición consensuada de términos especialmente conflictivos, debido a la importancia que tiene la definición de determinados conceptos para el avance de la ciencia (v.g. gen, luz, fuerza). Por ello, no debe resultar extraño que los investigadores en el área de la resolución de problemas encuentren dificultades a la hora de definir lo que es un problema o lo que implica la resolución de un problema.

\section{¿Qué significa el término problema dentro del marco de la enseñanza de la biología?}

Algunos autores definen el término problema como «una situación estimulante para la cual el individuo no tiene respuesta, es decir, el problema surge cuando el individuo no puede responder inmediata y eficazmente a la situación» (Woods et al. 1985). Considerar el problema como una situación que presenta dificultades para las cuales no hay soluciones evidentes, parece ser un acuerdo general entre los que han abordado el tema (GiI et al. 1988).

En un intento de combinar la tradición gestaltiana (por ejemplo, de los trabajos de Duncker (1945) con la tradición asociacionista (por ejemplo de los trabajos de $\mathrm{Ne}$ well y Simon 1972 o de Scandura 1977), Garrett (1986) define el problema como «situaciones donde el para- digma existente no puede aplicarse e incluso puede no existir solución, aquellas situaciones donde se conoce o asume que pueden resolverse con un paradigma dado recibirían la denominación de puzzle». Bajoesta concepción, el problema constituye un proceso productivo mientras que el puzzle corresponde a una situación cuya solución se alcanzará con un procedimiento meramente reproductivo.

Por otro lado, Garrett (1986) mantiene que «cada individuo, dependiendo de su conocimiento personal, personalidad y de las estrategias o recursos de que disponga, verá una situación dada como un problema o como un puzzle». De este modo, el que una situación pueda considerarse o no problema es totalmente subjetivo.

Gil et al. (1988) consideran que para un profesor las situaciones planteadas en el aula como problemas (de lápiz y papel) no son tales problemas puesto que conoce su solución. Sin duda, esto es cierto considerado desde un punto de vista psicológico, que cuando una persona encuentra la forma de resolver un problema éste deja de existir.

Sin embargo, en el contexto del aula el profesor sigue hablando de problemas tanto si sabe o no resolverlos. Es más, sólo cuando ha resuelto un problema sabe si es o no adecuado para ser utilizado como herramienta de acceso al conocimiento. El profesor basa la elección de una determinada situación (problema), como forma de facilitar la comprensión, en las características del proceso de resolución de esta situación. Por ello, pensamos que basar una definición en un hecho totalmente subjetivo puede resultar extremadamente ambiguo, originando conflictos de difícil solución, y que en el contexto educativo el problema, como elemento de una estrategia de enseñanza, debe đefinirse en sí mismo, por las etapas que comporta su proceso de resolución, y no por la complejidad que presente para la persona que afronta tal situación.

Además, en la enseñanza de la biología necesitamos differenciar claramente lo que es una cuestión de lo que es un problema. Ambas son formas de exponer una duda pero ¿en qué se diferencian?, ¿qué caracteriza al problema? Por ejemplo:

En el guisante de jardín, Mendel encontró que la semilla amarilla era dominante a la semilla verde $(A>a)$ y que la forma redonda de la semilla era dominante a la forma rugosa $(\mathrm{R}>\mathrm{r})$. ¿Qué proporción fenotípica podemos esperar en F2 de una cruza de amarillo redondo $x$ verde rugoso?

Este ejemplo suele aparecer como problema en los libros de biología. Pero, ¿una situación que puede resolverse utilizando únicamente algoritmos o la memoria, puede considerarse un problema?, ¿podemos utilizar este tipo de situaciones como punto de partida de una estrategia de enseñanza basada en la resolución de problemas?

Probablemente, algunas personas no conocen medios o caminos evidentes de obtener la respuesta del ejemplo citado. Woods et al. (1985) considerarían esta situación como un problema para esas personas. Sin embargo, este tipo de planteamiento puede resolverse utilizando el 
significado de los términos fenotipo y dominante, almacenados en nuestra memoria, y haciendo uso de un simple algoritmo. El algoritmo ha sido definido como una «prescripción ya hecha, completamente determinada, de la forma de actuar' (Landa 1972). Lochhead y Collura (1981) añadieron que los algoritmos pueden ser «cajas negras utilizadas para producir respuestas con o sin comprensión». Por analogía podríamos considerar algo. ritmo la reproducción de un diagrama que puede haber aparecido en el texto o en el encerado durante la resolución de un determinado "problema» en clase. Smith (1988) mantiene que «des̀arrollar la habilidad o capaci. dad para reproducir un patrón y hacerlo apropiadamente puede ser, en efecto, aprendizaje, pero la realización o ejecución de la labor no es resolución de problemas». Efectivamente, este tipo de situaciones no debe conside. rarse un problema si no requiere análisis de los hechos y razonamiento para elaborar la estrategia a seguir durante el proceso de resolución, es decir, para diseñar la forma de obtener los datos necesarios (numéricos o no) y de procesarlos para conseguir la respuesta correcta, factores que en último término determinan la comprensión del contenido implícito (recordemos que el objetivo de la estrategia de resolución de problemas en la enseñanza de las ciencias es facilitar la comprensión).

Por otro lado, si para alcanzar la solución se requiere la selección o la integración de dos o más algoritmos, tal situación podría considerarse como un verdadero pro. blema. La elección del algoritmo apropiado que debe ser utilizado y la determinación de la secuencia en que los resultados de una primera etapa han de ser utilizados en la resolución de las siguientes, requiere del citado análisis y razonamiento. De hecho, la selección del algoritmo apropiado para resolver una situación cuya solución se alcanza utilizando uno solo, también implica un juicio y un cierto grado de comprensión. Ésta es una forma de reconocer y elegir una estrategia o un patrón de resolución que puede considerarse como un componente de la resolución de problemas. Pero, estas dificultades no implican la creación de una estrategia de resolución, por lo tanto, son únicamente ejercicios de reconocimiento. Además, si al alumno se le pide que resuelva el problema en clase, en muchos casos, el estudiante supone que el problema propuesto se resuelve utilizando ese algoritmo. Así, el alumno no realizará ningún tipo de selección. Por lo tanto, Ia situación no será resuelta como un problema.

En resumen, pensamos que el problema en el contexto del aula y como componente base de una estrategia de enseñanza basada en su resolución puede definirse como una situación cuya solución requiere que el sujeto analice unos hechos y desarrolle razonadamente una estrategia que le permita obtener unos datos (numéricos o no), procesar estos datos (relacionarlos entre sí y con los hechos), interpretarlos y llegar a una conclusión (respuesta). Este análisis y razonamiento debe basarse en la comprensión del tema o del campo al que pertenece la situación. Un problema no podrá ser resuelto mediante el recuerdo, el reconocimiento, la reproducción o la aplicación de un único algoritmo. De este modo el problema vendrá definido por el proceso đe resolución que deberá seguir la persona que intenta alcanzar su solución y no por el grado de dificultad que presente para esa persona.

\section{¿Qué es la resolución de problemas?}

Frazer (1982) considera que la resolución de problemas es un proceso que utiliza el conocimiento de una disciplina (en su caso la Química) y las técnicas y habilidades de esa disciplina para salvar el espacio existente entre el problema y su solución. Efectivamente, la resolución de problemas prodría concebirse como un proceso que conlleva una serie de actividades cuyo fin es la consecución de la solución. Tal definición resultaría aplicable a cualquier disciplina de las ciencias experimentales y estaría de acuerdo con la concepción deweyana del término. Sin embargo, el modelo de definición no establecería las condiciones internas que se desarrollan en el sujeto que resuelve el problema. En este sentido, la psicología gestaltiana concibe invariablemente la resolución de problemas como un proceso "productivo", donde el sujeto requiere de un período de «incubación» seguido de una repentina «intuición» mediante la cual reorganiza mentalmente la estructura del problema (Mayer 1977).

No obstante, existen concepciones diferentes. Kempa (1986) considera la resolución de problemas como un procesado de información que tiene lugar en el cerebro del sujeto y que implica y requiere varias funciones de su memoria (memoria a corto plazo, memoria de trabajo y memoria a largo plazo). Según este modelo, el estudiante lee el problema y lo interpreta en términos de tareas que se solicitan e ideas fundamentales que se requieren. A continuación el estudiante selecciona los métodos, estrategias y hechos que pueden conducirle a la solución. Por lo tanto, el sujeto ha de haber comprendido el problema y lo que en él se plantea pero no conoce de manera inmediata Ia estrategia que le conducirá a la solución, habrá de seleccionar ésta entre un conjunto de ellas. Éste es, probablemente, uno de los puntos más controvertidos del proceso de resolución de problemas que además hemos incluido en el significado de la palabra «problema».

En el área de las Matemáticas, Polya (1968) considera la resolución de problemas como un proceso de aprendizaje así como un objetivo en sí mismo y como una técnica básica que es necesario desartollar. Aunque podríamos analizar estos tres enfoques, nos limitaremos en este artículo a considerar sólo uno de ellos, la resolución de problemas como proceso de enseñanza y de aprendizaje.

\section{¿Qué tipos de problemas podemos plantear en la ense- ñanza de la biología?}

Siguiendo el sistema de clasificación propuesto por Frazer (1982) podemos diferenciar problemas «artificiales» y problemas «reales». El problema artificial será aquél cuya solución es conocida por la persona que lo ha presentado (el profesor o el autor del libro de texto). Este tipo de problemas puede clasificarse en función de la naturaleza de la solución, como:

a) Problema cerrado, con una solución única. Por ejemplo: 
En Drosophila un gen $R$ que es dominante y ligado al sexo determina que el ojo se reduzca y estreche por lo que se llama "ranurado", y el tipo común de ojo es determinado por el alelo recesivo $r$. Una hembra homocigótica tipo común es apareada con un macho con ojo ranurado. Determine las expectaciones genotipicas de los machos de la segunda generación filial.

La única solución posible es $1 / 2 \mathrm{r} / \mathrm{Y}$ tipo común, $1 / 2 \mathrm{R} /$ Y ojo ranurado.

b) Problema abierto, con un número variable de soluciones. Por ejemplo:

El siguiente pedigrimuestra la herencia de la sordera en el hombre. Los símbolos abiertos representan audición normal y los símbolos sólidos la sordera. Indique los genotipos de cada miembro de este pedigrí hasta donde sea posible, usando los simbolos $A, a$ y $B, b$.

Esquema $n^{2} /$

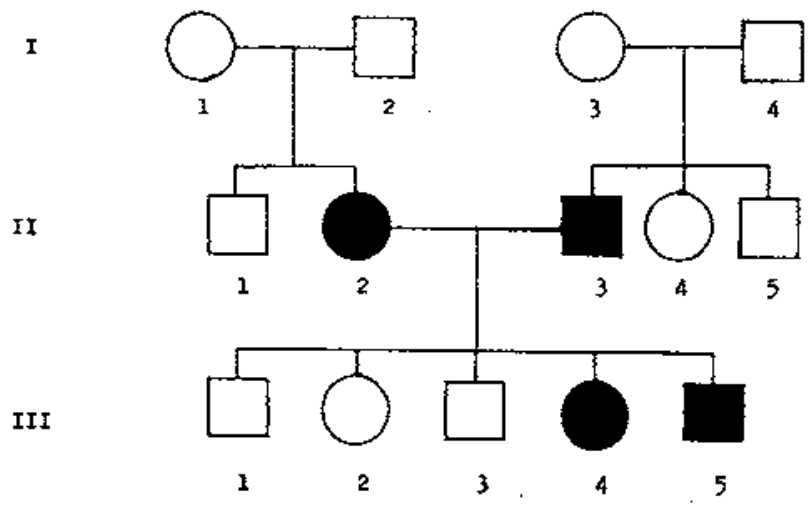

En este caso son posibles dos juegos de soluciones: juego $n^{\circ} 1$ : A-Bb(I1,2), AaB-(I3,4), A-B-(II1,4,5), $\mathrm{Aabb}(I I 2), \mathrm{AaBb}(I I 3), \mathrm{AaBb}(I I I 1,2,3)$, aa-o-bb(III4,5); juego $\mathrm{n}^{\mathrm{9}}$ 2: AaB-(I1,2), A-bb(13,4), A-B-(IIII 4,5$)$, aaBb(II2), Aabb(II3), AaBb(III1,2,3), aa-o-bb(III4,5).

En contraposición con el problema «artificial», el problema «real» será aquél donde no se conoce la solución, incluso puede que ésta no exista. Por ejemplo:

¿Cómo podríamos eliminar un tumor de estómago inoperable, utilizando rayos para destruir el tejido, evitando la destrucción de los tejidos sanos que rodean el tumor del paciente?

Problema planteado por Duncker (1945) para explicar las soluciones funcionales de tipo «árbol» y que en el momento en que fue afrontado no se conocía su solución.

Frazer (1982) diferencia, además, problemas reales con o sin objetivo dirigido, en función de que estén o no encaminados a resolver algún aspecto concreto de interés científico, tecnológico o social. La resolución de problemas reales requiere comportamientos adicionales sucesivos y algunas veces comportamientos en conjunto diferentes.

En la enseñanza de las ciencias, en general, los problemas utilizados son de tipo artificial y, salvo raras excepciones, cerrados. Este tipo de problemas se usan con dos propósitos: 1) facilitar la comprension al estudiante haciéndole utilizar su propio conocimiento y 2) prepararle para la resolución de problemas reales.

\section{COMPONENTES DE LA ESTRATEGIA DE ENSENANZA}

\section{Definición del problema}

En muchos casos, los problemas son definidos exclusivamente por el profesor o bien su enunciado figura en un libro de texto. Sin embargo, en otras ocasiones el alumno, dirigido por el profesor, puede participar en la definición del problema. A nuestro juicio, una estrategia de enseñanza basada en la resolución de problemas debecontemplar la redefinición del problema en el aula. Actualmente, de hecho, existen programas de educación general básica que contemplan la definición del problema y la resolución de éste como componentes básicos de la estrategia de enseñanza de las ciencias experimentales. Por ejemplo, el programa «Elementary Sciencie Syllabus», desarrollado por el Departamento de Educación del estado de New York para impartir enseñanzas en el area de ciencias desde la edad de 4 años, propone el modelo esquematizado en la fig. 1 .

El problema es definido a través de un proceso que se inicia partiendo de una experiencia y que concluye con la formulación de unas preguntas. La respuesta a estas preguntas se alcanzará al finalizar el proceso de resolıción. "Poner a los alumnos en situación de aplicar la metodología científica -es decir, de emitir hipótesis, de diseñar experimentos, de realizarlos y analizar con rigor los resultados- se convierte así en una necesidad tanto para la superación de «errores conceptuales»-es decir, para hacer posibles los profundos cambios conceptuales que ello implica - como para entender, en general, los resultados del trabajo científico» (Gil 1983).

Para facilitar la comprensión del proceso seguido hasta la formulación de las preguntas podemos analizar la siguiente situación, planteada en el aula a alumnos de sexto de EGB:

Sobre una mesa se colocaron tres vasos llenos de agua. Cada uno tenía un embudo invertido debajo del cual se habian colocado algunos brotes de Elodea canadiensis. Acoplados al extremo del embudo teníamos unos tubos calibrados, invertidos y llenos de agua. El primer vaso se iluminó con una lámpara de $100 \mathrm{~W}$ situada a $25 \mathrm{~cm}$ de distancia. El segundo vaso se iluminó con una lámpara similar situada a $100 \mathrm{~cm}$ de distancia. Por último, el tercer vaso se recubrió totalmente con un papel negro. Además, junto a los vasos colocamos una botella con 
una solución de $\mathrm{NaHCO}_{3}$ (la experiencia se preparó una hora antes de comenzar la clase).

Los alumnos observaron que existía un desprendimiento de burbujas de gas en las plantas situadas debajo del embudo, en aquellos vasos que estaban iluminados y además que este gas se acumulaba bajo los tubos calibrados, los cuales contenían diferentes volúmenes de gas. Seguidamente se originó una discusión en grupos del fenómeno observado, to cual sirve de apoyo a la formulación de preguntas y por lo tanto a la definición del problema por parte de los alumnos. Tal como afirma Ausubel (1978), «la discusión es el método más eficaz y realmente el único factible de promover el desenvolvimiento intelectual con respecto a los aspectos menos bien establecidos y más controvertidos de la materia de estudio».

Las discrepancias propias de las discusiones en grupo dieron lugar a la formulación de algunas preguntas como: ¿qué proceso tiene lugar en cada vaso?, ¿qué clase de gas se recoge en cada tubo?, ¿por qué hay diferencias en el volumen de gas recogido en cada tubo? ¿qué efecto tiene la separación de la fuente de luz sobre el proceso observado? A estas preguntas el profesor añadió otras como: $i$ alguno de los tres vasos corresponde a un experimento control?, ¿cómo podríamos comprobar la clase de gas recogido en los tubos?, ¿por qué usamos plantas acuáticas en esta experiencia?

Alcanzado este punto habremos definido el problema. Para comprenderlo debemos lograr responder a todas las preguntas planteadas.

E1 mismo problema podría presentarse verbalmente de la siguiente forma:

Al iluminar brotes de Elodea canadiensis, sumergidos en agua, con una lámpara de $100 \mathrm{~W}$ situada a 25 y 100 cm de distancia se abservó que el volumen de gas desprendido erade 1 y 0,2 ccrespectivamente. Fragmentos similares de la citada planta mantenidos en oscuridad no desprendian gas. ¿A qué se deben las diferencias observadas?, ¿qué clase de gas se desprende?, ¿cómo puede identificarse el gas desprendido?

En este caso el alumno no aprende a definir el problema, se limitará a buscar respuesta a las preguntas planteadas. En contraposición con la presentación anterior del problema, este planteamiento limita la participación y la espontaneidad del alumno y el protagonismo del alumno en el aula (favoreciendo el protagonismo del maestro). El descubrimiento activo es sustituido por la transmisión verbal o escrita de conocimientos teóricos y abstractos, la experimentación es sustituida por la teoría, la actividad por el sedentarismo, el derecho a la individualidad de pensamiento por la uniformidad de la medida y la creatividad por la imitación de arquetipos. Recordemos que el objetivo de la enseñanza no consiste simplemente en instruir al alumno y darle unos modelos de conducta, sino en desarroliar al máximo todas sus aptitudes y capacidades, en fomentar su interés por el mundo que le rodea, en infundirle autonomía, responsabilidad y sentido crítico.

\section{Resolución del problema}

El modelo de resolución planteado en la fig. 1, aunque puede ser aplicado por los alumnos desde los cuatro años de edad, resulta especialmente apropiado en el período piagetiano de «operaciones concretas» (de siete a once años de edad). Superada esta etapa, la estructura cogni. tiva del alumno permite el uso de modelos de destrezas y la experiencia de cada uno influirán en la estrategia de

\section{figural}

Modelo esquematizado de Definición y Resolución de Problemas.

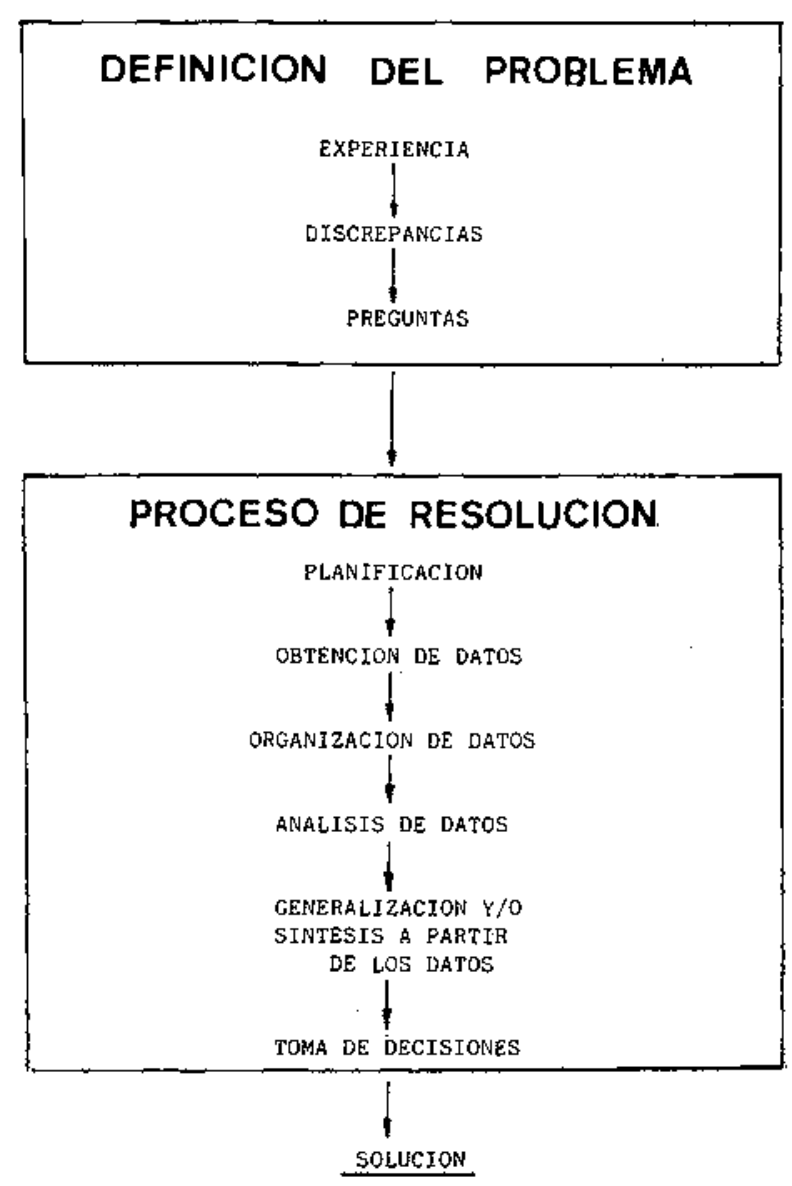

resolución, surgiendo en ellos distintas formas de alcanzar la solución. Murphy y Gott (1984) han analizado el proceso de resolución seguido por alumnos de 13 y 15 años de edad respectivamente y han identificado una serie de etapas comunes en el proceso, que les ha llevado a la construcción del modelo cíclico de resolución representado en la fig. 2. No obstante, algunos de los alumnos considerados resolvían los problemas de forma aparentemente lineal. El modelo cíclico es un proceso reiterativo e implica que el alumno realiza una evaluación, a distintos niveles, durante la búsqueda de la 
figura 2

Modelo cíclico de resolución de problemas.

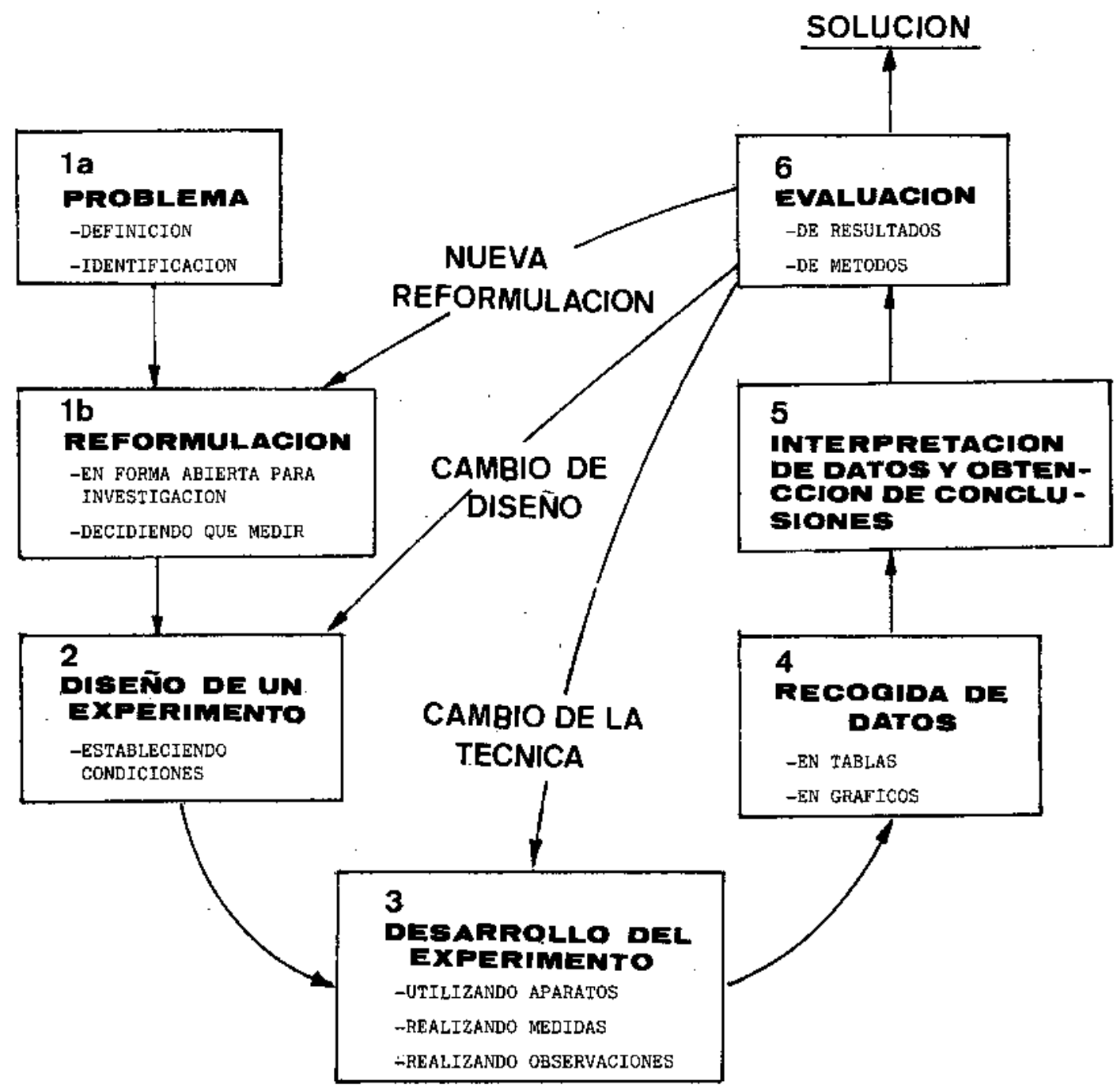

solución. Esto le permite rechazar sus conclusiones y tomar nuevas decisiones. El modelo aproxima el proceso de investigación científica a la resolución de problemas en el aula contemplando el aprendizaje como un proceso discontinuo de construcción y comprobación de hipótesis. En función de las hipótesis comprobadas, la persona que resuelve el problema intenta crear una secuencia de actuaciones que seguirá hasta que ésta resulte inapropiada para lograr la solución. La secuencia creada mediará entre el estímulo (el problema) y la respuesta (la solución). Esta visión del aprendizaje se opone a la concepción asociacionista de la teoría de la continuidad, pudiendo resultar apropiado para describir el proceso de pensamiento que tiene lugar en el período piagetiano de «operaciones formales» (Trabasso y Bower 1968, Mayer 1977).
Cuando el alumno inicia el proceso de resolución de un problema definido, crea un «espacio problema» interno, es decir una comprensión personal del problema (Newell y Simon 1972). Esta fase del proceso implica la reformulación del problema en unos términos en los cuales el sujeto comprende, estableciendo interrelaciones entre el problema y su conocimiento personal, incluyendo las similitudes existentes con problemas previamente resueltos y extractando los componentes más importantes del problema como vía para facilitar la selección y ejecución de los siguientes pasos (Hinsley, Hayes y Simon 1977). El reconocimiento de problemas similares es particularmente valioso, puesto que proporciona la puesta en marcha de experiencias y procedimientos conocidos (hechos, algoritmos y heurísticas) que serán la guía de la etapa de resolución subsiguiente (Chi, 
Feltovitch y Glaser 1981). Algunos aspectos de esta fase (fig. 2, ib) se han denominado «redescripción», puesto que la persona a menudo abstrae hechos notables del problema y los describe de una forma diferente. La mayoría de los ejemplos en este sentido están relacionados con la resolución de problemas en fúsica (Larkin et al. 1980), aunque también existen observaciones en el área de la genética que apoyan este hecho (Streibel et al., 1987). El desarollo de un espacio problema inicial propicio depende claramente deI uso adecuado de una base de conocimiento propia.

La planificación de la estrategia de resolución lieva asociadas cuestiones como qué información poseo acerca del problema?, ¿qué nueva información necesito?, ¿qué secuencia de acciones debo seguir?, ¿cómo puedo encontrar lo que necesito saber acerca de...?, ¿cómo saber cuando he resuelto el problema? La información que el alumno posee acerca del problema estará almacenada en su memoria semántica. La utilidad de la información dependerá de cómo ésta haya sido organizada y de cómo pueda ser recuperada para afrontar las diversas tareas. En este sentido, la práctica común de la enseñanza de las ciencias naturales y de la Biología en España ha puesto demasiada poca atención en la consideración de la organización del conocimiento. Los contenidos de los programas son generalmente presentados al alumno secuencialmente, capítulo por capítulo, de forma que los mismos estudiantes deben, de alguna forma, intentar integrar todos Ios conocimientos acumulados y organizarlos coherentemente para facilitar su uso (la tarea de organizar de forma eficaz el conocimiento implica una dificultad substancial para la que la mayoría de los estudiantes de EGB y BUP están mal preparados). Reif (1981) ha propuesto un sistema de organización del conocimiento universalmente aplicable, basado en la descomposición de cualquier dominio de conocimiento en «bloques de conocimiento» jerárquicamente organizados. De este modo, un bloque de primer orden contendrá la información de los hechos más relevantes del dominio entero; un pequeño número de ideas de este bloque de conocimiento serân después perfiladas añadiendo información en bloques de conocimiento subordinados, y así sucesivamente. La organización de cualquier conocimiento enseñado no es menos importante que sus contenidos. Por ello, uno debe procurar que este conocimiento sea presentado en una forma efectivamente organizada y que éste resuite útilmente organizado en la mente del alumno.

Una vez elaborada la secuencia de pasos a seguir para lograr la solución, la obtención de datos constituirá una parte integrante de cualquier modelo de resolución. En esta etapa, el alumno se plantea la pregunta ¿qué nueva información necesito? Podrá necesitar datos cualitativos o cuantitativos. El proceso de obtención de datos dependerá en gran medida del uso de los sentidos. La validez de los datos obtenidos dependerá del grado de exactitud y precisión ejercido por la persona que los obtiene.

Una vez conseguida la información necesaria, ésta se organiza. El alumno establece algunos patrones para ordenar o integrar los datos obtenidos buscando respuesta a la pregunta ¿cómo puedo organizar la información de forma útil? La forma elegida por el alumno será considerada provisional o temporal. Si đurante el posterior desarrolto del proceso de resolución el alumno descubre que la forma de organización elegida no es adecuada necesitará cambiarla o incluso modificar o desechar la estrategia de resolucion inicial.

El siguiente paso tanto en el modelo de la fig. 1 (analizando datos) como en el de la fig. 2 (interpretando datos) requiere que el alumno establezca las relaciones entre Ios datos, reconozca las características que proporcionan Ias relaciones causa-efecto y haga constar las deduciones posibles. El análisiss de los datos debe ser cuidadoso y razonado para asegurar una resolución eficaz. Este análisis Ileva al alumno a obtener conclusiones o a elegir alternativas posibles que le lleven a la solución.

Por último, la toma de decisiones y la evaluación completan el proceso de resolución. Esta etapa final Ileva asociadas preguntas como: ¿qué decisiones debo tomar?, ¿cuáles han sido las alternativas elegidas y por qué?, ¿cuáles son las consecuencias de cada alternativa?, ¿el proceso seguido ha sido correcto?, ¿la solución obtenida tiene sentido?, etc. Si no se ha logrado la solución, la evaluación del proceso permitirá deducir el camino a seguir decidiendo la validez de cada uno de los pasos seguidos durante la resolución.

\section{A MODO DE CONCLUSIÓN}

Para terminar resumiremos los aspectos que consideramos más importantes en la resolución de problemas como parte integrante de la estrategia de enseñanza de la biología:

La resolución de problemas supone una concepción dinámica de la educación basada en la comprensión.

En el marco de la enseñanza de la biología, el problema podría definirse como una situación cuya solución requiere que el sujeto analice unos hechos y desarrolle razonadamente una estrategia que le permita obtener unos datos, procesarlos, interpretarlos y llegar a una conclusión.

La resolución del problema es un proceso basado en la comprensión del área de conocimiento del que se ha extraído el problema. Éste no podrá ser resuelto mediante el recuerdo, el reconocimiento, la reproducción o la aplicación de un único algoritmo.

El modelo de resolución deberá instruir al alumno de forma que sea capaz de emitir hipótesis y de siseñar estrategias o experiencias para su corroboración. La comprobación de la solución constituirá la fase final del proceso.

La estrategia de enseñanza de las ciencias naturales basada en la resolución de problemas debería contemplar la definición de una experiencia o situación natural. 


\section{REFERENCIAS BIBLIOGRÁFICAS}

AUSUBEL, D.P., 1978. Psicología Educativa. Un punto de vista cognoscitivo. (Trillas: México).

CHI, M.T.H., FELTOVITCH P.J.' y GLASER R., 1981. Categorization and representation of physics problems by experts and novices, Cognitive Science, 5, pp. 121-152.

DEWEY, J., 1975. Experiences in Education. (New York Collier Books: New York).

DUNCKER, K., 1945. On problem solving, Psychological Monographs, 58, p. 5, Número 270.

ELEMENTARY SCIENCE SYLLABUS, 1985. (The State Education Department, Division of Program Development: New York State, Univ. Albany, New York).

FRAZER, M.J., 1982. Solving Chemical Problems, Chemical Society Review, 11(2), pp. 171-190.

GARRETT, R.M., 1986. Issues in science education: problem solving creativity and originality, International Journal Science Education, 9(2), pp. 125-137.

GIL, D., 1983. Tres paradigmas básicos en la enseñanza de las Ciencias, Enseñanza de las Ciencias, 1, pp. 26-33.

GIL, D. y MARTÍNEZ-TORREGROSA J., 1986. La reșolución de problemas como instrumento de cambio metodológico, Educación Abierta, 66, pp. 31-59.

GIL, D. y MARTINEZ-TORREGROSA J., 1987. La resolución de problemas de Fisica. (Ediciones del MEC: Madrid, Vicens Vives: Barcelona).

GIL, D., MARTÍNEZ-TORREGROSA J. y SENENT, F., 1987. Fracaso en la resolución de problemas de Física: una investigación orientada por nuevos supuestos, Investigación y experiencias didácticas, $6(2)$, pp. 131-147.

GIL, D., DUMAS, A., CAILLOT, M., MARTÍNEZ-TORREGROSA, J. y RAMÍREZ, L., 1988. La resolución de problemas de lápiz y papel como actividad de investígación, Investigación en la Escuela, 6, pp. 3-19.

HARLEN, W., 1983. Science at age 11. (Science report for teacher, DES: Londres.)

HEALEY, J.A.D., 1980. Distillation as a practical problem, School Science Review, 61, pp. 464-475.

HINSLEY, D.A., HAYES, J.R. y SIMON, H.A., 1977. From words to equations: meaning and representation in algebre word problems, en Just, M.A. y Carpenter, P.A. (eds.), Cognitive processes in comprehension. (Lawrence Erlbaum Associates, Hillsdale: New Jersey).

JOHNSTONE, A.H. y KELLETT, N.C., 1980. Learning Difficulties in School Science-Towards a Working Hypothesis, European Journal Science Education, 2(2), pp. 175-181.
KEMPA, R.F., 1986. Resolución de problemas de química y estructura cognoscitiva, Enseñanza de las Ciencias, 4(2), pp. 99-110.

LANDA, L.N., 1972. Algorithmization in learning and instruction. (Educational Technology Publications, Englewood Cliffs: New Jersey).

LARKIN, J.H., HELLER, J.I. y GREENO J.G., 1980. Instructional implications of research on problem solving, $\mathrm{New}$ Directions for Teaching and Learning, 2, pp. 5\-65.

LOCHHEAD, J., COLLURA, J., 1981. A curse for cookbook laboratories, The physics teacher, 19, pp. 46-50.

MASKILL, R. y WALLIS, K.G., 1982. Scientific thinking in the clasroom, School Science Review, 63, pp. 551-554.

MAYER, R.E., 1977. Thinking and Problem-Solving: An Introduction to Human Cognition and Learning. (Scott, Foresman and Company Glenview: Illinois).

MURPHY, P. y GOTT, R., 1984. The Assesment Framework at 13715. (Science Report for Teacher, DES: Londres).

NEWELL, A. y SIMON, H.A., 1972. Human problems solving. (Prentice Hall, Englewood Cliffs: New Jersey).

PHILPOT, A. y SELLWOOD, P., 1983. An introduction to problem-solving activities-some suggestions for design and make, School Science Review, 65, pp. 489-500.

POLYA, G., 1968. Mathematical discovery Vol. II: On understanding, learning and teaching problem solving. (Wiley: New york).

REIF, $F+, 1981$. Teaching problem solving - A scientific approach, The physics teacher, 19(5), pp. 310-316.

SCANDURA, J.M., 1977. Problem Solving: A Structuralt Process Approach with Instructional Implications. (Academic Press: New York).

SMITH, M.U., 1988. Toward a unified theory of problemsolving: a view from biology. Annual Meeting of American Educational Research Association, New Orleans, USA.

STENHOUSE, L., 1987. La investigación como base de la enseñanza. (Morata S.S.: Madrid).

STREIBEL, M.J., STEWARD, J., KOEDINGER, K., COLLINS A. y JUNGCK, J.R., 1987. Mendel: An intelligent computer toturing system for genetics problem-solving, conjeturing and understanding, Machine Mediated Learning, 2 , pp. $129-159$.

TRABASSO, T.R. y BOWER, G.H., 1968. Attention in learning. (Wiley: New York).

WOODS, D.R., CROWE, C.M., HOFFMAN, T.W. y WRIGHT, J.D., 1985. Challenges to teaching problem-solving skills, Chem. 13 News (Waterloo University), 155, pp. 1-12. 